\title{
Agregação de Tráfego e Imparcialidade em Redes Ópticas WDM: Análise Utilizando Teoria de Grafos
}

\author{
Eduardo J. Aloia, Amílcar C. César e Murilo A. Romero
}

\begin{abstract}
Resumo- O objetivo deste artigo é analisar a agregação de tráfego em redes ópticas WDM. Para isto, um grafo genérico para a análise de redes heterogêneas em malha, baseadas no modelo de pares (peer model) foi elaborado. Em seguida, comparamos o desempenho de duas políticas de agregação dinâmica de tráfego: minimização da rota na topologia virtual (MrTV) e minimização da rota na topologia física (MrTF). Adicionalmente, visando melhorar o QoS da rede, um controle de admissão de chamadas (call admission control-CAC) foi avaliado. Embora bem sucedido, o CAC é uma solução de compromisso, por ocasionar em contrapartida um aumento da probabilidade bloqueio das conexões requisitando menor largura de faixa.
\end{abstract}

Palavras-chave - Rede óptica WDM, imparcialidade, agregação, tráfego dinâmico, roteamento, alocação de recursos, otimização.

\begin{abstract}
The goal of this paper is to analyze traffic grooming aspects of WDM optical networks. To this aim, we constructed a generic graph for the analysis of heterogeneous meshed networks, based on the peer model. Next, we compared the performance of two distinct dynamic traffic grooming policies: MrTV, which uses the shortest path in virtual topology, and MrTF, which uses the shortest path in physical topology. In addition, we also implemented and evaluated the performance of a call admission control (CAC) mechanism in order to improve the network QoS. Although successful, such CAC imposes a trade-off because it imposes a raise on the blocking probability for low-bandwidth call requests.
\end{abstract}

Keywords - WDM networks, fairness, dynamic traffic grooming, optimization, resource assignment.

\section{INTRODUÇÃO}

Nos últimos anos, o rápido crescimento do número de usuários e o aumento da utilização dos serviços baseados na Internet (aplicações de voz, vídeo e multimídia) impulsionaram a necessidade de maior largura de faixa. Redes ópticas WDM (wavelength division multiplexing) surgem como tecnologia-chave neste cenário.

Eduardo J. Aloia, Amílcar C. César e Murilo A. Romero, Universidade de São Paulo, Escola de Engenharia de São Carlos, Depto. Eng. Elétrica, São Carlos, SP. E-mails: \{edujoal;amilcar;muriloa\}@sel.eesc.usp.br.

Esta pesquisa foi parcialmente financiada pelo $\mathrm{CNPq}$ (bolsas produtividade em pesquisa), CAPES (bolsa de estudos de doutorado), FAPESP (projeto Tidia-KyaTera, proc. 03/08269-7) e CPqD-FINEPFUNTTEL (Projeto Giga, contrato no. 1360).
Esta infra-estrutura de rede apresenta marcante diferença entre a largura de faixa de um canal óptico (OC-48, OC-192) e a largura de faixa de uma conexão típica (STS-1, OC-3, OC-12). Por exemplo, os requisitos de largura de faixa de uma residência podem ser estimados em $100 \mathrm{Mbps}$ [1]. Neste sentido, busca-se agregar tráfego de menor largura de faixa em canais ópticos, de maneira eficiente em termos de utilização dos recursos, dando origem a uma nova funcionalidade na rede, denominada agregação de tráfego (traffic grooming).

Como será preciso agregar solicitações de conexão requerendo diversos valores de largura de faixa, aquelas que solicitam larguras de faixa maiores tendem a exibir probabilidade de bloqueio mais elevada do que as que requerem valores menores. Portanto, podem-se equilibrar as probabilidades de bloqueio das diversas solicitações. Este processo é conhecido por imparcialidade (fairness) e a sua adoção via controle de admissão de chamadas (call admission control-CAC) geralmente eleva o valor da probabilidade de bloqueio global das redes [2]. No entanto, esta solução de compromisso beneficia o tráfego que exige maior largura de faixa.

O problema de agregação de tráfego consiste em combinar tráfego requisitando diferentes larguras de faixa em um comprimento de onda. A alocação das larguras de faixa é feita em combinação com o roteamento do tráfego, conectando nó de origem e destino.

Assim, o problema pode ser formulado da seguinte maneira [3]. Seja uma configuração de rede incluindo topologia física, número de transmissores e receptores em cada nó, número de comprimentos de onda em cada fibra e capacidade de faixa disponível nos comprimentos de onda; seja o conjunto de solicitação de conexões com diferentes "granularidades" (larguras de faixa). A solução consiste em configurar os caminhos ópticos para satisfazer tais solicitações, utilizando o menor número possível de recursos de rede e minimizando a probabilidade de bloqueio para as futuras conexões.

Operacionalmente, é necessário dotar a camada IP (Internet protocol) de novas funcionalidades, incluindo engenharia de tráfego. Uma solução possível é aquela baseada no uso da tecnologia GMPLS (generalized multiprotocol label switching) [4]. A arquitetura GMPLS visa prover as redes ópticas de um plano de controle, derivado do MPLS (multiprotocol label switching). Para isto, algumas 
modificações e adições são necessárias para que os protocolos de sinalização e roteamento presentes no MPLS se adaptem às peculiaridades da comutação óptica. Desta maneira, o GMPLS é estendido para incluir um grupo de elementos de redes que não tomam decisões de comutação e roteamento baseados nas informações carregadas nos cabeçalhos de pacotes ou células, mas sim com base em timeslots, comprimentos de onda ou portas. Em outras palavras, a arquitetura de um plano de controle único proporcionado pelo GMPLS assegura a troca de rótulos (labels), enviados de uma porta de entrada para uma porta de saída, em qualquer tipo de nó da rede. Para roteadores IP, os rótulos designam principalmente portas de entrada e saída. Para redes ópticas, os rótulos designam portas de entrada e saída e comprimentos de onda ou faixa de comprimentos de onda para cada OXC (optical crossconnect). Para dispositivos de multiplexação por divisão de tempo, equipamentos SONET/SDH (synchronous optical network; synchronous digital hierarchy), os rótulos designam timeslots de entrada e saída [5].

Estas arquiteturas multicamadas podem ser gerenciadas separadamente-modelo coberto (overlay), ou conjuntamente - modelo de pares (peer). No caso do modelo coberto, tanto o roteamento dos caminhos ópticos sobre a topologia física quanto o roteamento das conexões sobre a topologia virtual são gerenciados por dois planos de controle. Portanto, a camada cliente GMPLS, formada por roteadores que comutam rótulos (label switching routers-LSRs), conhece somente os canais virtuais fornecidos pela topologia física. Desta forma, a estrutura interna da topologia física é invisível à camada cliente. No caso do modelo de pares, as duas camadas são gerenciadas por um plano de controle, que dispõe de todas as informações sobre as duas camadas. Desta forma, as decisões de roteamento dos canais ópticos e das conexões são consideradas de forma única.

Dependendo do modelo, existem duas abordagens para o problema de agregação de tráfego. Para o modelo coberto [6], [7], a decisão de roteamento é considerada independente nas duas camadas, pois cada uma delas tem seu próprio algoritmo de roteamento. Especificamente, o problema de estabelecimento de caminhos ópticos na topologia física é abordado como um problema de roteamento e alocação de comprimento de onda (routing and wavelength assignment RWA). Para o modelo de pares (peer) [8], [9] um algoritmo único de agregação de tráfego é necessário no plano de controle para fornecer caminhos ópticos e rotear as conexões.

Este artigo aborda a construção de um grafo auxiliar para modelo de pares. O compartilhamento de informações sobre o estado da rede entre as duas camadas proporciona melhor uso dos recursos globais da rede, enquanto o modelo coberto leva à subutilização dos recursos [6]. Este grafo, baseado em proposta de Zhu e Mukherjee [9], propicia a análise de diferentes formas de composição da topologia da rede, contemplando vários fatores de heterogeneidade, como número de transmissores e receptores em cada nó, número de comprimentos de ondas em cada fibra e a capacidade de conversão de comprimentos de onda e de agregação em cada nó. Embora este modelo de grafo possa ser utilizado para agregação de tráfego estático ou dinâmico, somente este último tipo será abordado neste artigo, por se tratar de um comportamento mais realista da rede. Além disso, vários algoritmos de agregação descritos na literatura já contemplam a forma estática [10].

A contribuição do artigo está em utilizar a técnica de gráfico auxiliar para analisar agregação de tráfego e imparcialidade de capacidade de largura de faixa em redes GMPLS que empregam o modelo de pares.

$\mathrm{O}$ artigo está organizado da seguinte forma. A Seção II descreve o modelo de rede. Na Seção III a construção do grafo é especificada. O algoritmo de funcionamento do grafo está descrito na Seção IV, onde duas políticas de agregação de tráfego foram estudadas: minimização da rota na topologia virtual (MrTV), utilizada para carregar o tráfego das conexões solicitadas usando os caminhos mais curtos na topologia virtual e minimização da rota na topologia física (MrTF), utilizada para rotear o tráfego através dos enlaces mais curtos na topologia física. Observou-se que as conexões requerendo larguras de faixa elevadas apresentam maiores probabilidades de bloqueio, evidenciando a parcialidade da rede. Com o objetivo de minorar este problema, não resolvido em [9], a Seção V descreve e analisa um controle de admissão de chamadas baseado na abordagem proposta por Thiagarajan e Somani [2]. A Seção VI descreve os resultados numéricos obtidos.

\section{MODELO DE REDE}

A topologia de rede utilizada é formada por duas camadas: a virtual e a física, como mostra a Fig. 1. Os roteadores IP/GMPLS, LSRs, estão situados no plano virtual e os OXC, no plano físico. Um caminho óptico (lightpath) entre os nós fonte e destino é estabelecido por um ou mais enlaces de fibras ópticas, interconectando OXCs. Cada enlace é constituído de um comprimento de onda.

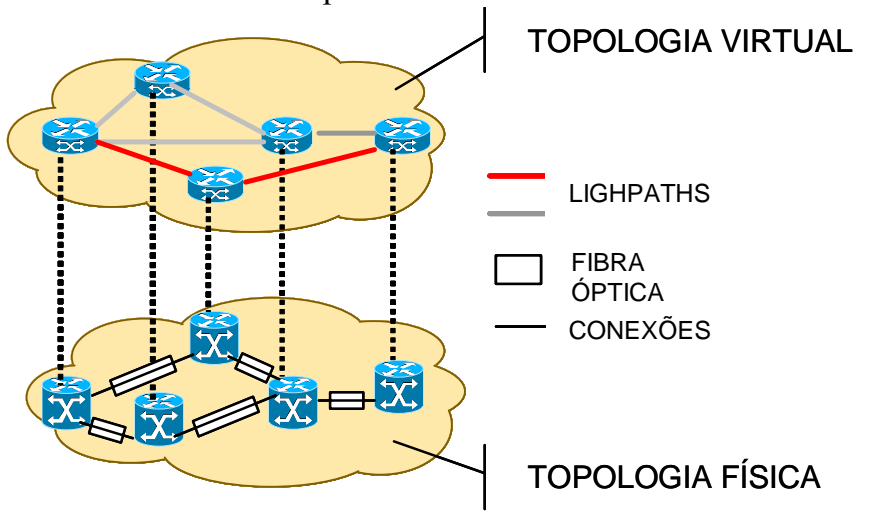

Fig.1. Topologia física e virtual em uma rede óptica.

As conexões "vêem" apenas a topologia virtual, sendo que uma ligação entre dois LSRs nesta topologia não necessariamente corresponde a uma ligação direta entre os respectivos $\mathrm{OXCs}$ na topologia física. 


\section{CONSTRUÇÃO DO GRAFO}

Para exemplificar o funcionamento da estrutura de rede formada pelo grafo auxiliar, é mostrada na Fig. 2 uma rede formada por três nós e dois comprimentos de onda. Em geral, uma rede pode ser representada pelo grafo $G_{0}\left(V_{0}, E_{0}\right)$, no qual $V_{0}$ e $E_{0}$ representam o conjunto de nós e enlaces, respectivamente.

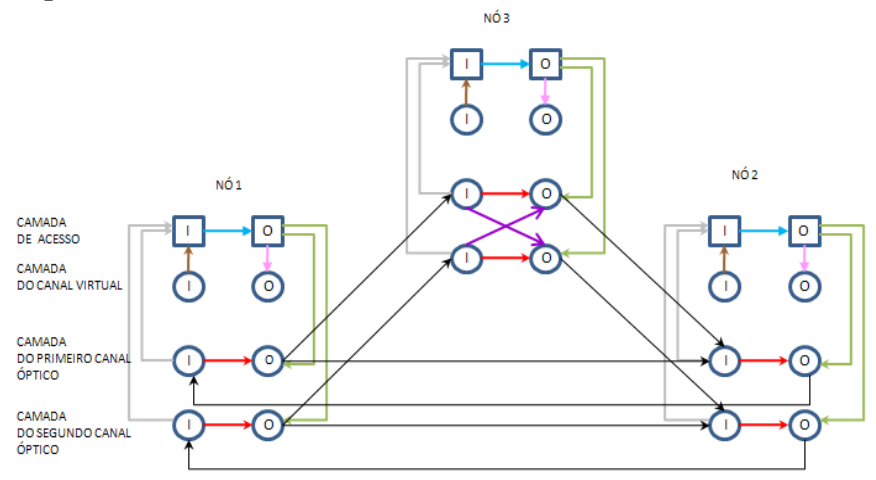

Fig.2: Grafo auxiliar para uma rede com três nós e dois comprimentos de onda.

O grafo auxiliar $\mathrm{G}(\mathrm{V}, \mathrm{E})$ empregará o termo vértices e arestas para o conjunto $\mathrm{V}$ e E. Na Fig. 2, cada quadrado ou círculo com a letra maiúscula "I" denota a porta de entrada em cada camada e o quadrado ou círculo com a letra "O" denota a porta de saída. O nó 3 está habilitado para conversão completa de comprimento de onda enquanto os demais não apresentam esta funcionalidade, evidenciada pela presença de arestas roxas se cruzando entre as camadas do primeiro e segundo comprimento de onda. O grafo auxiliar é composto por $W+2$ camadas, sendo $W$ o número de comprimentos de onda. As camadas 1 a $\mathrm{W}$ denotam as camadas dos comprimentos de onda, a camada do canal virtual é denominada camada da topologia virtual. É nesta camada que os enlaces entre os LSRs são configurados, ou seja, um enlace entre o nó 1 e 2 não apresentará necessariamente uma ligação na camada física (camada dos comprimentos de onda) exclusivamente entre os dois nós. As arestas são inseridas no grafo de acordo com:

1. Aresta de desvio de comprimento de onda (AdC). Esta aresta conecta a porta de entrada à porta de saída de cada camada de comprimento de onda. A capacidade desta aresta é considerada ilimitada e sua presença é representada pela cor vermelha;

2. Aresta de agregação (AdA). Esta aresta conecta a porta de entrada à porta de saída da camada de acesso, se o referido nó tem a funcionalidade de agregação. A capacidade desta aresta é considerada ilimitada e sua presença é representada pela cor azul;

3. Aresta de multiplexação (AdM). Esta aresta conecta a porta de saída da camada de acesso à porta de saída da camada do canal virtual em cada nó. A capacidade desta aresta é considerada ilimitada e sua presença é representada pela cor rosa;

4. Aresta de demultiplexação (AdD). Esta aresta conecta a porta de entrada da camada do canal virtual à porta de entrada da camada de acesso em cada nó. A capacidade desta aresta é considerada ilimitada e sua presença é representada pela cor marrom;

5. Aresta de transmissão (AdT). Esta aresta conecta a porta de saída da camada de acesso à porta de saída da camada de comprimento de onda se existe transmissor disponível no comprimento de onda $\lambda_{t}$ no nó $i$. A capacidade desta aresta é considerada ilimitada e sua presença é representada pela cor verde;

6. Aresta de recepção (AdR). Esta aresta conecta a porta de entrada da camada de comprimento de onda à porta de entrada da camada de acesso se existe receptor disponível no comprimento de onda $\lambda_{t}$ no nó $i$. A capacidade desta aresta é considerada ilimitada e sua presença é representada pela cor cinza;

7. Aresta de conversão (AcC). Esta aresta conecta a porta de entrada da camada de comprimento de onda L1 para a porta de saída de camada de comprimento de onda L2 no nó $i$, se o comprimento de onda $\lambda_{\mathrm{L} 1}$ pode ser convertido no comprimento de onda $\lambda_{\mathrm{L} 2}$. A capacidade desta aresta é tomada como ilimitada e sua presença é representada pela cor roxa;

8. Aresta de ligação de comprimentos de onda (AlC). Esta aresta conecta a porta de saída de cada camada de comprimento de onda $\mathrm{L}$ no nó $i$ à porta de entrada da camada de comprimento de onda $\mathrm{L}$ no nó $j$ se existe um enlace físico entre os dois nós e o comprimento de onda $\lambda_{t}$ deste enlace não esta sendo utilizado. A capacidade desta aresta é a capacidade inerente a cada comprimento de onda e sua presença é representada pela cor preta;

9. Aresta do canal virtual $(\mathrm{AcV})$. Esta aresta conecta a porta de saída da camada do canal virtual no nó $i$ à porta de entrada da camada do canal virtual no nó $j$ se existe um enlace virtual entre o nó $i$ e o nó $j$. A capacidade desta aresta é a capacidade residual do correspondente enlace virtual conectando o nó $i$ para o nó $j$. Nesta camada representa-se a topologia virtual e, portanto, não consta na Fig. 2.

O passo final na construção de um grafo auxiliar, não mostrado na Fig. 2, é a designação de pesos para as referidas arestas. Os pesos podem refletir o custo de cada elemento de rede (como transmissores, comprimentos de onda, conversores de comprimento de onda) e ou uma determinada política de agregação. Tais pesos podem ser fixos ou podem variar de acordo com o estado da rede. Neste sentido, o grafo auxiliar reflete o estado atual da rede. A rede pode ser heterogênea, com nós equipados com diferentes recursos e capacidades.

\section{DESCRIÇÃO DO GRAFO}

Um algoritmo de agregação de tráfego dinâmico [9] foi empregado baseado no grafo auxiliar mostrado na Fig. 2, 
cujas etapas são:

1. Construção do grafo auxiliar de acordo com o estado da rede;

2. Quando uma conexão T chega:

a. Compute o caminho mais curto entre a porta de saída da camada de acesso do nó inicial e a porta de entrada da camada de acesso do nó final. Se tal caminho não existe, bloqueie a conexão. Caso contrário, siga para o passo 3;

3. Se o caminho contém enlaces na camada física, configure uma conexão entre os nós;

4. Roteie T entre as conexões preexistentes (conexões já estabelecidas na camada do canal virtual) ou entre as configuradas no passo 3;

5. Atualize o grafo G como segue:

a. Para cada nova conexão configurada, uma aresta deve ser inserida no grafo na camada do canal virtual conectando a porta de saída do nó inicial à porta de entrada do nó final;

b. Os comprimentos de onda pertencentes à camada dos canais ópticos utilizados pela conexão são removidos do grafo auxiliar;

c. Se não existem mais transmissores/receptores disponíveis no nó i as arestas AdT e AdR serão removidas do grafo, de modo que o nó i não poderá mais iniciar ou receber novas conexões, com exceção das que utilizarão as conexões já criadas;

d. Para as conexões implementadas na camada do canal virtual, transportando o tráfego associado à solicitação $T$, as capacidades residuais inerentes a cada uma delas serão decrescidas da quantidade exigida pela solicitação $T$;

6. Quando uma conexão termina:

a. Remova o tráfego da rede;

b. Remova as conexões que não carregam mais nenhuma solicitação;

c. Atualize o grafo auxiliar com as operações contrárias às executadas no passo 5.

\section{FUNCIONAMENTO DO CAC}

A imparcialidade de capacidade de largura de faixa é alcançada quando a probabilidade de bloqueio de "c" requisições de taxa de transmissão "b" for igual à probabilidade de bloqueio de "b" requisições de taxa de transmissão "c" [2]. Se $P_{c}$ for a probabilidade de bloqueio de uma requisição de classe- $c$ e $P_{b}$ for a probabilidade de bloqueio de uma requisição de classe- $b$, a seguinte condição deve ser satisfeita para que a imparcialidade de capacidade de largura de faixa seja alcançada [2]:

$$
1-\left(1-P_{c}\right)^{b}=1-\left(1-P_{b}\right)^{c}, \forall 1 \leq b, c \leq g,
$$

na qual $g$ é igual ao número de classes de diferentes "granularidades" implementadas na simulação.

O CAC deverá alcançar imparcialidade de capacidade largura de faixa e manter a probabilidade de bloqueio global em nível aceitável. Consideramos que uma solicitação não pode ser dividida em diferentes taxas de transmissão ou entre enlaces.

A probabilidade de bloqueio global da rede pode ser definida em termos da probabilidade de bloqueio por unidade de taxa de transmissão. Quando a imparcialidade é obtida, considerando taxa de transmissão unitária $(b=1)$, de acordo com (1), tem-se que

$$
P_{c}=1-\left(1-P_{1}\right)^{c},
$$

ou

$$
P_{1}=1-\sqrt[c]{1-P_{c}}
$$

Assim, utilizando (3) pode-se obter uma estimativa do valor de $P_{1}$ em função de $P_{c}$. Esta estimativa, $\hat{p}_{c}$, é a probabilidade de bloqueio por unidade de taxa de transmissão de uma classe de transmissão c ou probabilidade de bloqueio normalizada. A estimativa da probabilidade de bloqueio global por unidade de taxa de transmissão, $P$ é dada por

$$
\hat{P}=\sum_{i=1}^{g} P_{i} / \sum_{i=1}^{g} i \text {. }
$$

Geralmente, a imparcialidade estabelecida afeta mais as requisições de maior ou menor capacidades do que as requisições de capacidade intermediária. Uma estimativa aproximada pode ser obtida utilizando apenas a probabilidade de bloqueio das taxas de transmissão mais elevadas e mais baixas. A taxa de imparcialidade (fairness ratio-FR) é a relação entre a probabilidade de bloqueio por unidade de taxa de transmissão para a solicitação de maior taxa, $\hat{p}_{g}$, e a solicitação de menor taxa, $\hat{p}_{1}$. Assim [2],

$$
F R=\hat{P}_{g} / \hat{P}_{1},
$$

Se o valor de FR for maior que 1, o algoritmo favorece as solicitações com taxas maiores em relação àquelas com taxas menores e vice-versa. Se for próximo de 1 significa que o algoritmo consegue estabelecer a imparcialidade na rede. Desta forma, o CAC torna-se um conjunto de decisões a serem tomadas para estabelecer se uma solicitação de conexão será aceita ou rejeitada. O CAC pode ser usado conjuntamente com os esquemas de roteamento e alocação de comprimento de onda para estabelecer imparcialidade entre as diversas solicitações, causando, no entanto, um aumento na probabilidade de bloqueio global da rede. 


\section{RESULTADOS NUMÉRICOS}

A simulação foi realizada em um $\mathrm{PC}$ com processador Pentium IV de 2,8 GHz, e memória RAM de 2,0 GB, sendo o software codificado em linguagem $\mathrm{C}$. A rede utilizada na simulação é a NSFNet, mostrada na Fig. 3, constituída de 14 nós e 42 enlaces unidirecionais. As solicitações de conexão seguem processo de Poisson e são uniformemente distribuídas em todos os nós.

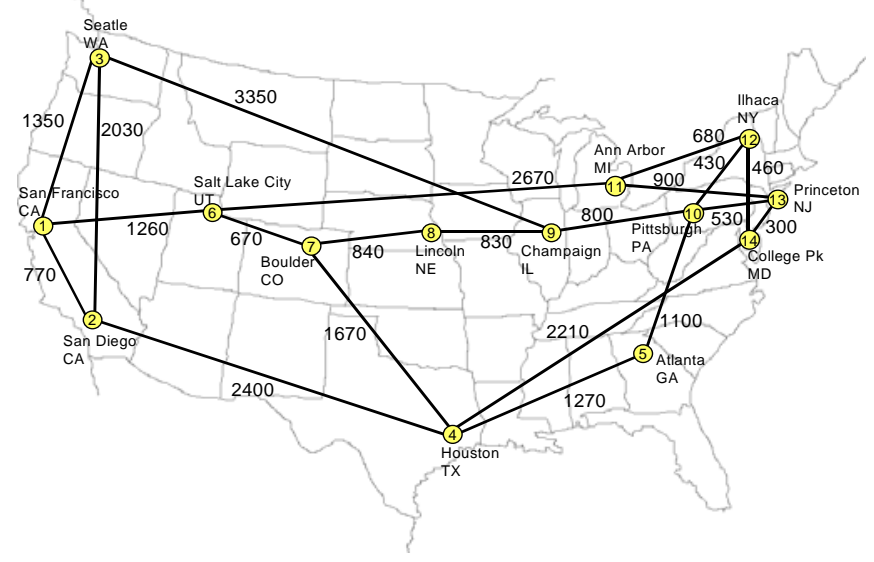

Fig. 3. Rede NSFnet.

O tempo de permanência das conexões segue uma distribuição exponencial com valor médio $1 / \mu=60 \mathrm{~s}$. A capacidade máxima de cada comprimento de onda é $10 \mathrm{Gbps}$. Cada solicitação de conexão pode dispor de taxa de transmissão $m, 1 \leq m \leq g$, com $g=4$, geradas com a seguinte probabilidade:

$$
R_{c}=(1 / c) / \sum_{m=1}^{g} 1 / m
$$

na qual $c=\{1,2,3,4\}$, respectivamente, para as conexões com taxa de transmissão $C B_{1}=2,5 \mathrm{Gbps}, C B_{2}=5,0 \mathrm{Gbps}$, $C B_{3}=7,5$ Gbps e $C B_{4}=10$ Gbps. As correspondentes probabilidades de bloqueio são, respectivamente, $48 \%, 24 \%$, $16 \%$ e $12 \%$. Para a rede analisada, cada nó possui 6 pares de transmissores e receptores, cada enlace unidirecional de fibra suporta 4 comprimentos de onda, todos os nós possuem capacidade de agregação (via conversão OEO) e nenhuma capacidade de conversão de comprimento de onda. A técnica de simulação por evento discreto é utilizada para gerar 100.000 solicitações de conexão. Os pesos para as arestas ditadas pelas duas políticas de agregação, MrTV e MrTF, estão especificados na Tabela 1.

A análise do gráfico da Fig. 4 mostra que a política MrTF produz uma probabilidade de bloqueio menor quando os transmissores não são o recurso mais restritivo. Tal resultado é confirmado por simulações com outras redes mais conectadas, não disponibilizadas aqui por falta de espaço. Assim, esta política pode ser aplicada à rede NSFnet para se analisar a probabilidade de bloqueio das diferentes "granularidades" de largura de faixa empregadas na simulação.
A Fig. 5 mostra a probabilidade de bloqueio para quatro diferentes "granularidades" de largura de faixa quando a rede NSFnet está submetida a uma carga de 60 erlangs.

TABELA 1: PESOS DESIGNADOS PARA CADA POLÍTICA DE AGREGAÇÃO

\begin{tabular}{|c|c|c|c|c|c|c|c|c|}
\hline Política & AlC & AdA & AdT & AdR & AcV & AdM & AdD & AdC \\
\hline MrTV & 10 & 1000 & 20 & 20 & 2 & 1 & 1 & 1 \\
\hline MrTF & 1000 & 1 & 20 & 20 & X & 1 & 1 & 1 \\
\hline
\end{tabular}

Nota-se que o peso designado à aresta $\mathrm{AcV}$ para a política MrTF é X, sendo, portanto, passível de alteração, pois ele varia de acordo com a quantidade de enlaces ópticos utilizados para a configuração do caminho óptico. Pode-se observar na Fig. 5 que as conexões requerendo larguras de faixa elevadas apresentam maiores probabilidades de bloqueio, ressaltando a parcialidade da rede. Visando alterar esta característica, utilizamos um CAC baseado em [2]. A imparcialidade estabelece a divisão da probabilidade de bloqueio normalizada da maior e da menor "granularidade" de largura de faixa, reduzindo a parcialidade da rede. Este mecanismo melhora o QoS da rede, proporcionada às conexões que requeiram as maiores taxas de transmissão. No entanto, há um aumento na probabilidade de bloqueio da rede, que passou de $2,83 \%$ para $5,18 \%$.

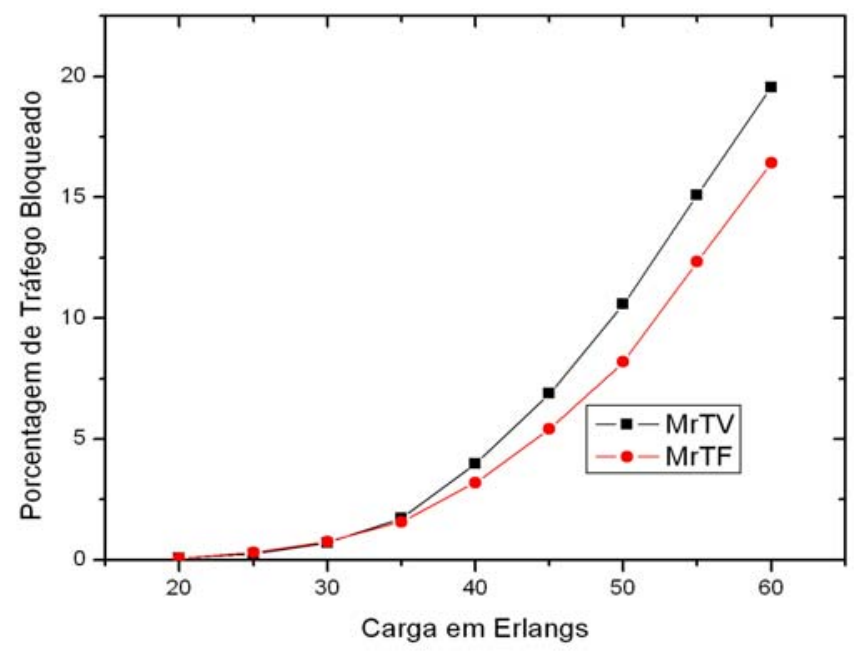

Fig.4: Probabilidade de bloqueio das políticas MrTV e MrTF na rede Nsfnet.

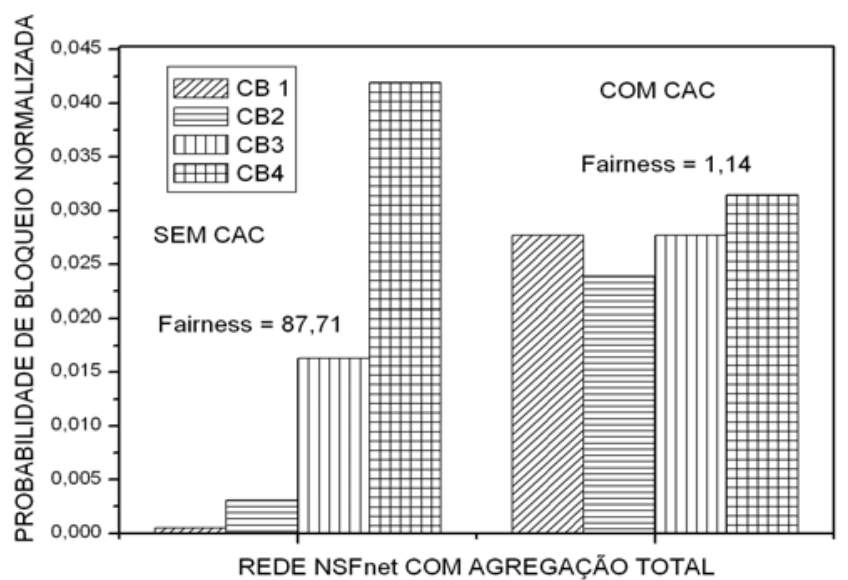


Fig.5: Probabilidade de bloqueio das diferentes larguras de faixa na rede NSFnet com carga de 60 erlangs.

\section{CONCLUSÕES}

Com objetivo de analisar políticas de agregação em tráfego dinâmico, um grafo genérico para a análise de redes heterogêneas em malha, utilizando o modelo de pares foi elaborado e analisado neste artigo. Foram adotadas duas políticas de agregação de tráfego. A MrTV, para transportar o tráfego das conexões pedidas usando o enlace mais curto na topologia virtual, e a MrTF, para transportar o tráfego através do enlace mais curto na topologia física.

A política de agregação MrTF apresenta menor probabilidade de tráfego bloqueado, ou seja, opera melhor em redes relativamente esparsas como a NSFnet, na qual a busca de um caminho alternativo torna-se mais difícil. Neste sentido, o número de comprimentos de onda disponíveis passa a ser o recurso mais restrito e a política de agregação que busca estabelecer os menores caminhos na topológica física apresenta melhor desempenho.

Como a política MrTF produz probabilidade de bloqueio menor quando os transmissores não são o recurso mais restritivo, esta política foi aplicada na rede NSFnet para se analisar a probabilidade de bloqueio de conexões com diferentes "granularidades" de largura de faixa. As conexões requerendo larguras de faixa elevadas apresentam maiores probabilidades de bloqueio, ressaltando a parcialidade da rede. Visando melhorar a QoS, um controle de admissão de chamadas foi utilizado. O CAC melhora o QoS da rede, resultando, porém, em aumento na probabilidade de bloqueio da rede.

\section{REFERÊNCIAS}

[1] M.J. O'Mahony et al., "Future Optical Networks". J. Lightwave Technol., v.24, n.12, pp.4684-4696, dezembro 2006.

[2] S. Thiagarajan e K.A. Somani, "Capacity Fairness of WDM Networks with Grooming Capabilities". Opt. Net. Mag., vol.2, n 3, pp. 24-31, maio/junho 2001.

[3] R. Dutta e G.N. Rouskas, "Traffic grooming in an optical WDM network: past and future”. IEEE Network Mag., vol. 16, $\mathrm{n}^{\circ}$ 1, pp.46-56, junho 2002.

[4] E. Mannie at al, "Generalized Multi-Protocol Label Switching (GMPLS) Architecture". Internet Engineering Task Force. IETF RFC 3945, October 2004. http://www.ietf.org..

[5] J.E. Aloia, "Sistematização Crítica das Tendências de Padronização de Arquiteturas e Protocolos em Redes Ópticas". Dissertação de mestrado. EESC-USP, 2003, $159 \mathrm{p}$.

[6] E. Salvadori, L.R. cigno e Z. Zsòka, "Dynamic grooming in IP networks base on the overlay architecture", Opt. Switch. Network., vol. 3, março 2006, pp. 118-133.

[7] S. Koo, G. Sabin e S. Subramaniam, "Dynamic LSP provisioning in overlay, augmented, and peer architectures for IP/MPLS over WDM networks", Proc. of INFOCOM 2004, pp. 7-11, Hong Kong, China, março 2004.

[8] W. Yao e B. Ramamurthy, "A link bundled auxiliary graph model for constrained dynamic traffic grooming in WDM mesh networks". ". IEEE J. Sel. Areas Commun., vol. 23, nº 8, pp.1543-1554, agosto 2005.

[9] H. Zhu, H. Zang e B. Mukherjee, "A novel generic graph model for traffic grooming in heterogeneous WDM mesh networks". IEEE ACM Trans. Network., vol. 11, n 2, pp. $285-299$, abril 2003.
[10] K. Zhu e B. Mukherjee, "Traffic grooming in an optical WDM mesh network," IEEE J. Select. Areas Commun., vol. 20, pp. 122-133, January 2002. 